\title{
The surface chemistry of near-infrared resonant gold nanotriangles obtained via thiosulfate synthesis
}

\author{
Maria Ana Huergo ${ }^{\mathrm{a}}$, , Lisandro Giovanetti ${ }^{\mathrm{a}}$, Aldo Alberto Rubert ${ }^{\mathrm{a}}$, Claudia Alejandra Grillo ${ }^{\mathrm{a}}$, \\ Mario Sergio Moreno ${ }^{b}$, Félix Gregorio Requejo ${ }^{a}$, Roberto Carlos Salvarezza ${ }^{a}$, Carolina Vericat ${ }^{\mathrm{a}}$ \\ a Instituto de Investigaciones Fisicoquímicas Teóricas y Aplicadas (INIFTA), Universidad Nacional de La Plata - CONICET, Sucursal 4 cc 16, 1900 La Plata, Argentina \\ ${ }^{\mathrm{b}}$ Centro Atómico Bariloche - 8400, S.C. de Bariloche, Argentina
}

\section{A R T I C L E I N F O}

\section{Keywords:}

Gold nanoparticles

Near infrared

Photothermal therapies

Thiosulfate

Reduced S species

\begin{abstract}
A B S T R A C T
Gold nanoparticles with NIR absorption are promising for photothermal therapy applications. Different syntheses have been proposed and, among them, that based on the reduction of Au(III) with thiosulfate is important because it yields gold nanotriangles (AuNTs) with strong absorption in the near-infrared region. It has been previously reported by others that the nanoparticle surface is covered by relatively weak adsorbed S species (mostly sulfate), which would render the surface easy to functionalize in order to improve biocompatibility and therapeutic effects. In this work we have used XPS, XANES, UV-vis-NIR spectroscopy, HRTEM and AFM to demonstrate that, in contrast to the previous reports, these AuNTs are covered by strongly adsorbed sulfur species (sulfide and polysulfide). A reaction pathway is proposed to explain the presence of reduced sulfur species and the absence of adsorbed sulfates and thiosulfates. Preliminary cytotoxicity assays show that the S-capped AuNTs do not show a deleterious effect for Au concentrations larger than those needed for in vivo photothermal treatments.
\end{abstract}

\section{Introduction}

Plasmonic gold nanoparticles (AuNPs) are of utmost interest in nanoscience due to their singular physico-chemical properties, many of them related to their localized surface plasmon resonance (LSPR), and play a key role for many nanotechnological applications such as sensing, electronic, optical devices, medical treatments and diagnostics [1-5]. This is even more so because of their chemical stability in different media and owing to the fact that they can be tailored in size and shape and post-functionalized to tune their surface properties and to immobilize different species of interest $[1,6]$.

In recent years much effort has been done to improve the synthesis of gold nanotriangles (AuNTs) because of the advantages related to their high anisotropy and the energy of its LSPR in the NIR region [7-10]. The optical and electrical properties of AuNTs are not only of interest in the field of basic plasmonics but also for their use as building blocks, metamaterials, templates and device components in sensors, as well as for nanomedical applications
[11-13]. AuNTs are a promising alternative to gold nanorods and nanoshells for plasmonic photothermal therapies [11].

Many different protocols have been proposed, including seeded-mediated syntheses in the presence of cationic surfactants (like CTAB and CTAC), some with halide and/or $\mathrm{Ag}^{+}$ions [8,9,14], syntheses with aminoacids as reductants [15], as well as with different natural products $[16-18]$ and vesicles $[19,20]$. Many of these have a low AuNT yield and give a mixture of nanoparticles of different shapes, making necessary a post-synthesis separation step. Also, it is still a challenge to produce AuNTs that absorb beyond $750 \mathrm{~nm}$ : this difficulty mostly resides in the fact that AuNTs with side length $<100 \mathrm{~nm}$ (the optimal size range for a successful targeting in cancer therapies due to the leaky vasculature) need to be thinner than $15 \mathrm{~nm}$ in order to absorb at such low energies [21].

An interesting strategy to overcome this is the use of sulfur compounds as reducing agents of $\mathrm{Au}(\mathrm{III})$ ions in aqueous solutions. Sulfide and thiosulfate have been both used to obtain truncated AuNTs with intense absorption in the so-called NIR-I biological window (around $800 \mathrm{~nm}$ ) [22-27]. However, due to the presence of spherical AuNPs in addition to the AuNTs, there has been some

Abbreviations: NIR, near-infrared; AuNPs, gold nanoparticles; AuNTs, gold nanotriangles; XPS, X-ray photoelectron spectroscopy; XANES, X-ray absorption near edge spectroscopy;

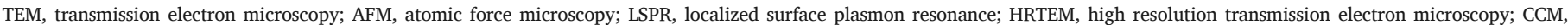
complete culture medium; AO, acridine orange; NR, neutral red; MTT, tetrazolium salt; FFT, Fast Fourier Transform; BE, binding energy.

* Corresponding author.

Email addresses: mahuergo@gmail.com (M.A. Huergo); lisandro@fisica.unlp.edu.ar (L. Giovanetti); rubert@quimica.unlp.edu.ar (A.A. Rubert); cgrillo@inifta.unlp.edu.ar (C.A. Grillo); smoreno@cab.cnea.gov.ar (M.S. Moreno); requejo@inifta.unlp.edu.ar (F.G. Requejo); robsalva@inifta.unlp.edu.ar (R.C. Salvarezza); cvericat@inifta.unlp.edu.ar (C. Vericat) 
controversy about the nanoparticles responsible for NIR absorbance [28-32]. Indeed, only recently it has been possible to thoroughly understand the optical and chemical properties of the AuNPs synthesized with sulfide, a fact that has somehow limited their applications $[21,33]$.

Of the two syntheses, that with thiosulfate has attracted greater interest because it is easier to achieve, has a larger yield of AuNTs compared to the case of sulfide and because it has been suggested that the nanostructures surface is covered by relatively weak adsorbed species that could be easily removed by straightforward ligand exchange processes [24,27]. In fact, the chemical reaction proposed by some authors involves the reduction of $\mathrm{Au}(\mathrm{III})$ to $\mathrm{Au}(0)$ by the thiosulfate ions that in turn oxidize to sulfates, which do not adsorb as strongly as other $\mathrm{S}$ species on gold surfaces $[24,27]$. However, the chemistry of thiosulfate, especially in acidic media, exhibits a great complexity, opening the possibility for alternative reaction pathways that can lead to a completely different chemistry. Indeed, it is widely accepted that thiosulfate decomposes in acidic media resulting in elemental sulfur, sulfur dioxide, hydrogen sulfide and polysulfanes, among other species [34].

The surface chemistry of thiosulfate on metallic gold also reveals a great complexity, and different adsorbed $\mathrm{S}$ species have been detected. Indeed, gold sulfide, $\mathrm{S}_{8}$, pyritic $\mathrm{S}_{2}{ }^{2-}$ and tetrathionate-like species have been detected by XPS for polished gold in thiosulfate solutions [35]. Also, STM studies show that the initial immersion of Au(1 11) substrates in thiosulfate solutions in alkaline media results in the formation of ordered adlayers of thiosulfate ions that can be reduced to yield sulfide species that adsorb as monomeric S [36]. Moreover, it is well-known that nanoparticles are more reactive than planar surfaces due to the large number of defects on their surface [37-39].

This opens new questions about the chemistry involved in the thiosulfate synthesis of AuNPs, not only concerning their bulk composition but also regarding the surface species present, especially if they are to be used for biomedical purposes [40-42]. In this work we present a thorough physico-chemical study of the synthesis of AuNTs resulting from the reaction of $\mathrm{Au}(\mathrm{III})$ with thiosulfate. Our results show that the AuNPs are covered by several strongly adsorbed reduced sulfur species, similar to those found when sulfide is used as the reductant [33]. This leads us to conclude that the thiosulfate synthesis proceeds by a mechanism that involves sulfide species as the reducing agent. Therefore, unlike what has been proposed by other authors, the route of thiosulfate oxidation to sulfate is not the only reducing pathway possible for $\mathrm{Au}(\mathrm{III})$ ion reduction $[24,27]$. The presence of reduced S species on the surface of the AuNTs is most challenging and must be taken into account if the nanoparticles are designed for nanomedical applications. In fact, these species will affect postfunctionalization of the AuNTs and could have some deleterious effect on some cellular processes [43,44]. Our preliminary in vitro studies show, however, that the S-capping does not show a detrimental effect on the studied fibroblast cell line.

\section{Materials and methods}

\subsection{Synthesis of gold nanoparticles}

A detailed description of the synthesis of AuNPs by reduction of $\mathrm{HAuCl}_{4}$ with $\mathrm{Na}_{2} \mathrm{~S}_{2} \mathrm{O}_{3}$ can be found elsewhere [27]. Briefly, $10 \mathrm{~mL}$ of $1.71 \mathrm{mM} \mathrm{HAuCl}_{4}$ (Sigma-Aldrich) solution were quickly mixed with $3.2 \mathrm{~mL}$ of $3 \mathrm{mM} \mathrm{Na}_{2} \mathrm{~S}_{2} \mathrm{O}_{3}$ (Sigma-Aldrich) solution at room temperature. The reaction was allowed to evolve, typically for $30-60 \mathrm{~min}$, and was then arrested at the selected position of the NIR peak by fast addition of $10 \mathrm{~mL}$ of the thiosulfate solution. This was done when the peak maximum reached a wavelength of approximately $800 \mathrm{~nm}$ during the blueshift. For TEM, S K XANES and some XPS measurements the arrested AuNPs were subsequently purified by centrifugation (10-30 min at $7000 \mathrm{rpm}$ ), followed by the replacement of the supernatant with ultrapure water.

The synthesis of the AuNPs prepared with $\mathrm{Na}_{2} \mathrm{~S}$ as reductant included for comparison has been previously described in Refs. [21,33] Purification was performed following the same steps.
The evolution of the syntheses and the stability of the obtained nanostructures were studied by UV-vis-NIR spectroscopy by means of a Lambda 35 spectrophotometer (Perkin Elmer).

\subsection{Transmission electron microscopy (TEM)}

Samples for transmission electron microscopy were prepared by dropping the aqueous dispersion on ultrathin carbon film supports (Ted Pella, Inc). Selected area electron diffraction patterns and HRTEM images were acquired using a Tecnai F20 G2 TEM operated at room temperature and $200 \mathrm{kV}$. Image analysis was done using the tools implemented in Digital Micrograph (Gatan Microscopy Suite ${ }^{\circledR}$ ).

\subsection{Atomic force microscopy (AFM)}

Measurements were performed in air in the intermittent (Tapping ${ }^{\mathrm{TM}}$ ) mode with a Multimode microscope operated by a Nanoscope V control unit, both from Veeco Instruments (now Bruker Corporation, USA). Silicon tips $\left(f_{o}=285 \mathrm{kHz} ; \mathrm{k}=42 \mathrm{~N} / \mathrm{m}\right)$ were used in all measurements and samples were prepared by drop-casting the dispersions onto freshly cleaved HOPG substrates (Ted Pella Inc.). Image analysis was done with Nanoscope 7.30 software.

\subsection{Au L3-edge X-ray absorption near edge spectroscopy (Au L3 XANES) experiments}

XANES experiments at the Au L3-edge $(11919 \mathrm{eV})$ were performed at the XAFS-2 beamline of the LNLS (Campinas, Brazil) which is equipped with a $\mathrm{Si}(111)$ double crystal monochromator. The X-ray beam is vertically and horizontally collimated giving a spot at the sample of about $0.2 \mathrm{~mm} \times 3 \mathrm{~mm}$. All experiments were performed in transmission mode adjusting the gas mixtures in the chambers for the required energy range. The energy calibration was obtained by measuring a thin Au film simultaneously with the sample, through a third ionization chamber. XAFS data were processed by standard methods using ATHENA software, which is part of the IFFEFIT package [45]. Samples were measured in solution using a sample holder consisting of plastic pieces, about $1 \mathrm{~cm}$ thick, sealed with Kapton ${ }^{\circledR}$ windows.

\subsection{S K-edge X-ray absorption near edge spectroscopy (S K XANES) experiments}

Experiments were performed at the SXS beamline at the LNLS (Campinas, Brazil) [46], which is equipped with a $\operatorname{InSb}(111)$ double crystal monochromator with slit aperture of $1 \mathrm{~mm}$, giving an energy resolution of about $0.6 \mathrm{eV}$. Sodium thiosulfate was used as reference for energy calibration by assigning the value $2481.5 \mathrm{eV}$ to the highest maximum of $\mathrm{Na}_{2} \mathrm{~S}_{2} \mathrm{O}_{3}$ (corresponding to the so-called inner sphere), in accordance with the criteria previously reported by Vairavamurthy [47]. All samples were prepared by drop casting on carbon disks (Ted Pella, Inc). All reported experiments in the $\mathrm{S} \mathrm{K}$ edge were measured in ultra-high vacuum (chamber pressure $<10^{-7}$ torr). Absorption spectra for each sample was recorded in the fluorescence mode following the intensity of $S \mathrm{~K} \alpha_{1,2}$ emission lines (2309.5 and $2308.4 \mathrm{eV}$, respectively).

\subsection{X-ray photoelectron spectroscopy (XPS)}

Measurements were done with a non-monochromatic $\mathrm{Al} \mathrm{K \alpha}$ source at $1486.6 \mathrm{eV}$ (XR50, Specs $\mathrm{GmbH}$ ) and a hemispherical electron energy analyzer (PHOIBOS 100, Specs $\mathrm{GmbH}$ ) operating either at 10 or $40 \mathrm{eV}$ pass energy. A two-point calibration of the energy scale was performed using sputtered cleaned gold ( $\mathrm{Au} 4 \mathrm{f}_{7 / 2}$, binding energy $=84.00 \mathrm{eV}$ ) and copper $\left(\mathrm{Cu} 2 \mathrm{p}_{3 / 2}\right.$, binding energy $\left.=932.67 \mathrm{eV}\right)$ samples. $\mathrm{C} 1 \mathrm{~s}$ at $285 \mathrm{eV}$ was used as charging reference. Samples were prepared by drop casting on highly oriented pyrolytic graphite (HOPG) substrates (Ted Pella, Inc). Purified (centrifuged) samples were carefully rinsed with water and dried with $\mathrm{N}_{2}$, while non-arrested, non-purified samples were only dried with $\mathrm{N}_{2}$. Spectra were analyzed with 
CasaXPS v2.3.14 software. Shirley-type backgrounds were used in the fitting procedure of high resolution spectra. For quantitative analysis, peak intensities were corrected by the corresponding Relative Sensitivity Factors. The fitting of the $S 2 p$ peaks was carried out by using a spin-orbit splitting of $1.18 \mathrm{eV}$ and a branching ratio of 0.5 .

\subsection{Biological assays}

Fibroblast cell line BALB/c 3T3 were used to evaluate the cytocompatibility of several concentrations of AuNPs. Cells were routinely cultured with DMEM culture medium (GIBCO) supplemented with $10 \%$ fetal calf serum (Natocor), $50 \mathrm{IU} / \mathrm{mL}$ penicillin and $50 \mu \mathrm{g} / \mathrm{mL}$ streptomycin sulfate $\mathrm{CCM}$ in a humidified incubator at $37^{\circ} \mathrm{C}$ and $5 \% \mathrm{CO}_{2}$ atmosphere.

Three sets of experiments were arranged in order to evaluate the effect of solutions of different concentrations AuNPs on BALB/c 3T3 cells: (1) adhesion and cell proliferation measured by epifluorescence microscopy after AO; (2) the NR assay was performed according to Borenfreud and Puerner to measure cellular transport based on the dye uptake by living cells [48]; and (3) MTT assay, as described by Mosmann and modified by Twentyman and Luscombe $[49,50]$, to measure the reduction of MTT to formazan by dehydrogenase enzymes of intact mitochondria in living cells.

For NR and MTT assays $2.5 \times 10^{3}$ cells/well were cultured in 96 multi-well plate and grown at $37^{\circ} \mathrm{C}$ in $5 \% \mathrm{CO}_{2}$ humid atmosphere in CCM for $24 \mathrm{~h}$. Then, the medium was replaced by other containing AuNPs. Different concentrations of AuNPs were used: 25, 50, 100, 200 and $500 \mu \mathrm{M}$, expressed as gold concentrations (which correspond to approximately 5, 10, 20, 40 and $100 \mu \mathrm{g} / \mathrm{mL}$ of Au). The stability of AuNPs in CCM was tested by UV-vis-NIR spectroscopy. After $24 \mathrm{~h}$ medium was removed and fresh medium containing $40 \mu \mathrm{g} / \mathrm{mL}$ NR dye (Sigma) or MTT reagent ( $1 \mathrm{mg} / \mathrm{mL}$ final concentration) (Sigma) was added. After $3 \mathrm{~h}$ incubation, cells were washed with a phosphate buffer saline solution (PBS). Color was developed by the addition of $0.1 \mathrm{~mL} 1 \%$ acetic acid in 50\% ethanol for NR assay and with $100 \mu \mathrm{L}$ dimethylsulfoxide (DMSO) (Merck) for MTT test. The plates were shaken for $10 \mathrm{~min}$ and the absorbance was measured (NR: $540 \mathrm{~nm} / \mathrm{MTT}$ : $560 \mathrm{~nm}$ ) using an automatic ELISA plate reader ( $\mu$ Quant BioTek). The absorbance change is directly proportional to the number of viable cells. Untreated cells were used as control. In order to evaluate the possible absorbance of AuNPs, a set of wells only with AuNPs (without cells) were run simultaneously with AuNPs-treated cultures. The cytotoxicity percentage was calculated as $[(A-B) / A] \times 100$, where $A$ and $B$ are the absorbances of control and treated cells, respectively. The absorbance average of AuNPs was subtracted from the absorption of the treated cells to obtain the B value. Each experiment was repeated in two independent sets, that is, a total of 24 wells were tested for each concentration. Data were analyzed using one-way ANOVA test and multiple comparisons were made using $\mathrm{p}$ values corrected using the Bonferroni method.

In order to assess the influence of the AuNPs treatments on cell adhesion and proliferation, $5 \times 10^{5}$ cells were seeded in 6 multi-well plate and grown at $37{ }^{\circ} \mathrm{C}$ in $5 \% \mathrm{CO}_{2}$ humid atmosphere in CCM. After $24 \mathrm{~h}$ medium was replaced by aliquots with different final concentrations of AuNPs (25, 50, 100, 200, 500 $\mu \mathrm{M}$ of gold) and under these conditions cells were cultured during $24 \mathrm{~h}$. Additionally, BALB/c $3 \mathrm{~T} 3 \mathrm{cell}$ cultures without AuNPs were also evaluated and used as control. After exposure time, cells were washed with PBS, adherent cells were stained with AO dye (Sigma) and immediately examined by epifluorescence microscopy (Olympus BX51) equipped with appropriate filter connected to an Olympus DP71 color video camera. Surface densities of cells were obtained from digital images using Image-Pro Plus program. Each assay was repeated twice in independent experiments.

\section{Results and discussion}

\subsection{Optical and structural characterization}

The UV-vis-NIR spectra of the synthesis of AuNPs with thiosul- deed, in both cases there are two peaks, one around $530 \mathrm{~nm}$ and a second one in the NIR region that rapidly shifts to larger wavelengths and then gradually returns to shorter wavelengths, as it can be seen in Fig. $1 \mathrm{~b}$ [21]. For both syntheses, the position of the NIR peak can be arrested by adding an excess of sulfide or thiosulfate solution, which allows us to tune its position without the need to change the reagents proportion.

A typical TEM image of the products of the synthesis with thiosulfate (after purification) is shown in Fig. 1c and evidences the presence of AuNTs with different degree of truncation and spherical-like AuNPs of different size. The same structures can be observed by AFM, and the cross section profiles of the different AuNPs (performed horizontally and in the center of each nanostructure) (Fig. 1d), confirm that the AuNTs are quasi 2D in shape (Fig. S1), while nanospheres are 3D. In all cases the observed structures are very similar to those obtained from the sulfide synthesis (Fig. S2)[33]. Fig. 1e shows the HRTEM image of a single spherical AuNP over an AuNT in plan view, while Fig. 1f shows the FFT of the area marked by the black square in Fig. 1e, revealing the so-called forbidden 1/3[422] reflections of metallic gold in a $\langle 111\rangle$ incidence, as already reported. Their origin has been attributed to the formation of stacking faults in the fcc lattice [51], and these can be observed in the image of a typical AuNT in side view (Fig. 1g). Nanoplatelets and nanorods are occasionally found in the images, and they account for $<3$ and $4 \%$ of the number of AuNTs, respectively (see Fig. S1).

In the case of the sulfide synthesis, while the UV-vis-NIR peak at $530 \mathrm{~nm}$ had been unequivocally assigned to the spherical AuNPs, that in the NIR had been a matter of controversy and recently our group has assigned it to the presence of AuNTs and interpreted the red and subsequent blueshift of this peak in terms of the structural changes of the AuNTs ([21] and references therein). The same correlation between the peaks in the extinction spectra and the structure of the different AuNPs can be now applied to the thiosulfate synthesis. When comparing both spectra in Fig. 1a, there is a noticeable difference in the intensity of the NIR vs the peak at $530 \mathrm{~nm}$, which clearly increases in the case of thiosulfate. This fact can be attributed to the proportion of the spherical AuNP and AuNT populations (Fig. 1). Indeed, a comparison of the fraction of AuNTs relative to the total number of AuNPs of the TEM and AFM images of both syntheses confirms that the use of thiosulfate as reductant results in a higher AuNT yield (see bar graph in the inset in Fig. 1a). In any case, to further increase the concentration of AuNTs for photothermal therapies it is desirable to separate both populations, for instance by a method based on depletion forces [9], an issue that is not the scope of this paper.

\subsection{XANES and XPS chemical analysis}

The thiosulfate synthesis is thus an interesting choice to easily obtain AuNTs with optimal optical properties with a higher yield compared to that using sulfide. However, at this point, a new question arises concerning the nature of the surface species present on these AuNPs, which will determine their interfacial properties.

As regards the sulfide synthesis, in a previous paper we concluded that the species involved in the gold reduction were reduced sulfur compounds, and that the surface of the different AuNPs was capped by sulfides (monomeric S), polysulfides and also some elemental $S$ [33]. In the case of the thiosulfate synthesis, however, the use of a reagent with a more complex chemistry in aqueous solutions and, especially, the fact that some authors studying AuNT syntheses similar to ours concluded that sulfate ions were the only surface species on the AuNPs [24,27], evidences the need of an in depth study of the nature of the surface species and of the possible reactions that occur in the thiosulfate synthesis. We have therefore performed XPS and XANES measurements in order to characterize the chemical nature of the products of the synthesis.

Fig. 2 shows the Au L3-edge XANES spectrum obtained for AuNPs arrested at $800 \mathrm{~nm}$ and purified by centrifugation (see the Experimental section). Spectra corresponding to a gold metallic foil and $\mathrm{Au}(\mathrm{I})$ and $\mathrm{Au}(\mathrm{III})$ reference compounds are included for comparison. A general inspection clearly indicates the predominant metallic state of the $\mathrm{Au}$ atoms present in the sample. This result, coincident with those obtained from HRTEM analysis, which only reveal 

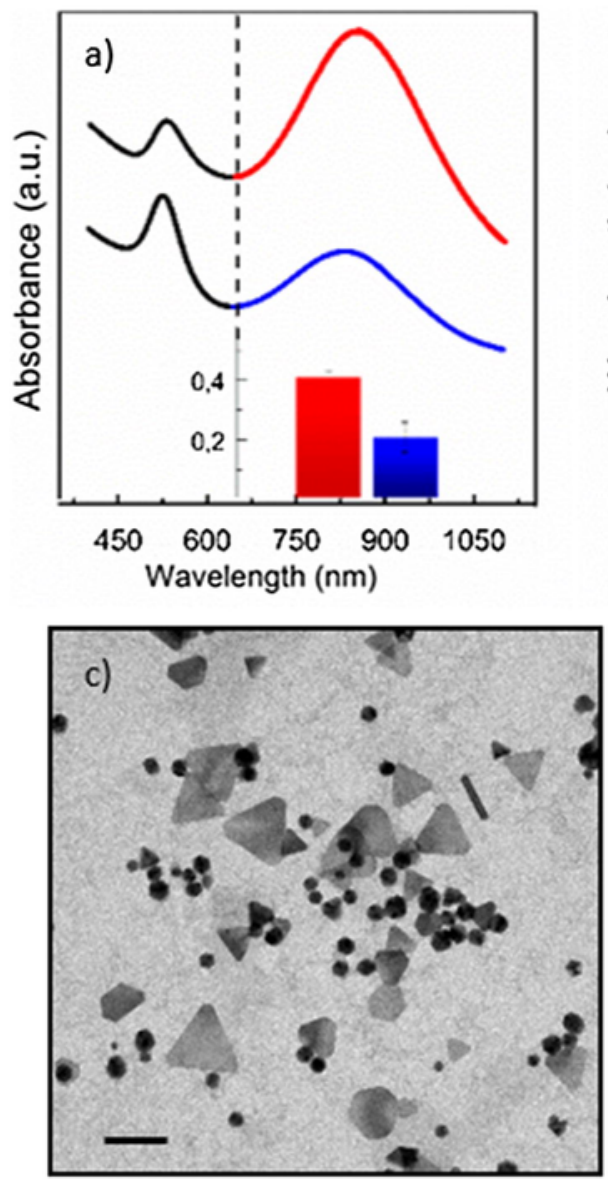
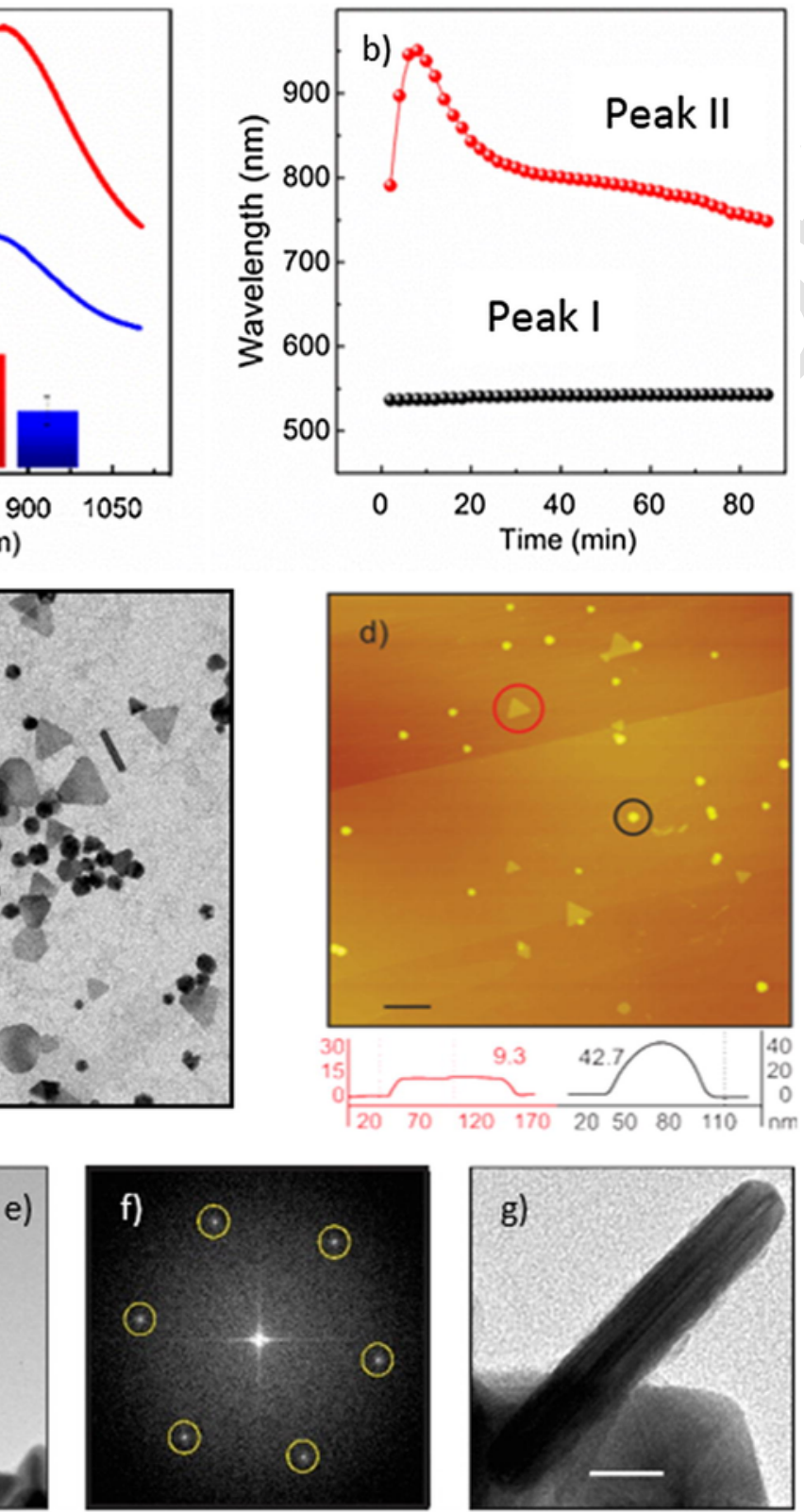

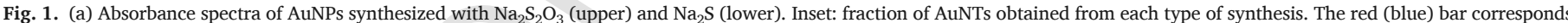

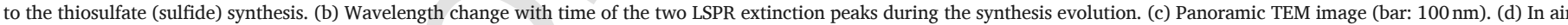

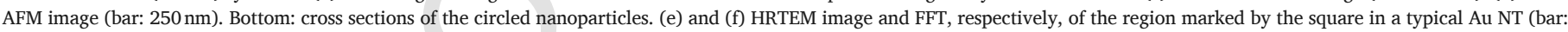

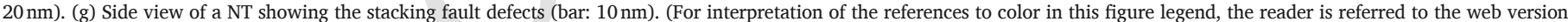
of this article.)

lattice distances corresponding to metallic gold, excludes the possibility that AuNPs synthesized by this route contain oxidized gold species [33]. Moreover, XPS spectra of the Au $4 \mathrm{f}$ region show only the component of metallic gold at $84.0 \mathrm{eV}$ (spectrum not shown).

As regards the $\mathrm{S}$ species, Fig. 2 shows XANES spectra at the $\mathrm{S}$ K-edge of the purified products of the thiosulfate and the sulfide synthesis (red and blue lines, respectively). The thiosulfate reference (black line in Fig. 2b) has two contributions at 2472.4 and $2481.5 \mathrm{eV}$, which correspond to the outer and inner sulfur atoms in thiosulfate, respectively [52]. The spectrum of the AuNPs from the thiosulfate synthesis presents three broad peaks, at about 2473, 2479.1 and $2483.5 \mathrm{eV}$. The lowest energy peak is assigned to reduced sulfur species (sulfides, polysulfides and elemental S) [33] and is similar to that observed in purified samples from the sulfide synthesis (blue line in Fig. 2b), while the other two are in the region corresponding to oxidized sulfur species and can be attributed to $\mathrm{SO}_{3}{ }^{2-}$ and $\mathrm{SO}_{4}{ }^{2-}$ species, respectively [47]. Noticeably, there is no evidence of thiosulfate species in these spectra. The presence of a sulfate peak in the spectra of the AuNPs from both syntheses can be attributed to the oxidation of the reduced sulfur species as a result of the sample preparation procedure [33]. The small peak assigned to sulfite species, only present in the spectrum of the thiosulfate synthesis, probably has the same origin. However, the presence of sulfate in the AuNPs resulting from the thiosulfate synthesis could also arise from the thiosulfate oxidation to reduce the $\mathrm{Au}(\mathrm{III})$ ions, i.e. the accepted reaction for this synthesis [24,27].

In order to gain more insight into the study of the $S$ species detected by XANES, especially those on the surface of the AuNPs, we have performed XPS measurements in the S 2p region. Fig. 3a shows the high resolution spectrum of a purified sample of AuNPs obtained from the thiosulfate synthesis. A broad $S 2 p$ peak is observed that can be fitted with three components, S1, S2 and S3, with $2 \mathrm{p}_{3 / 2}$ binding energies (BE) at $161.3,162.4$ and $164.2 \mathrm{eV}$, respectively, that correspond to different reduced $S$ species, as previously reported 


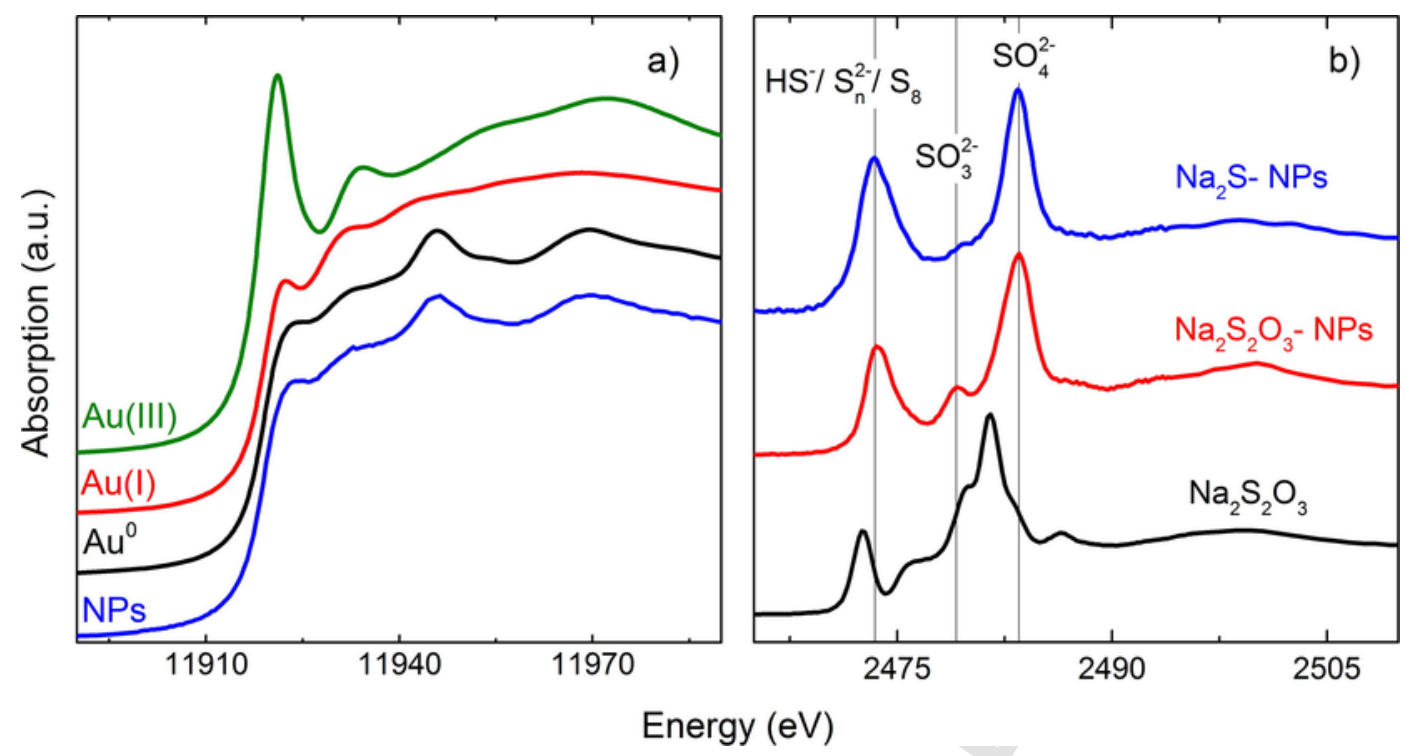

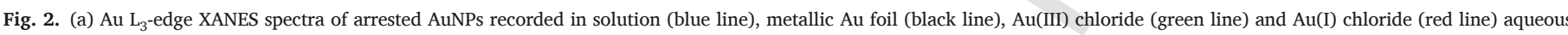

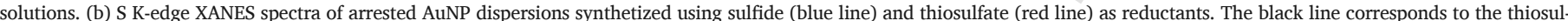

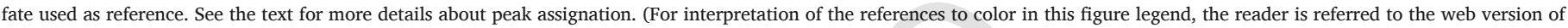
this article.)
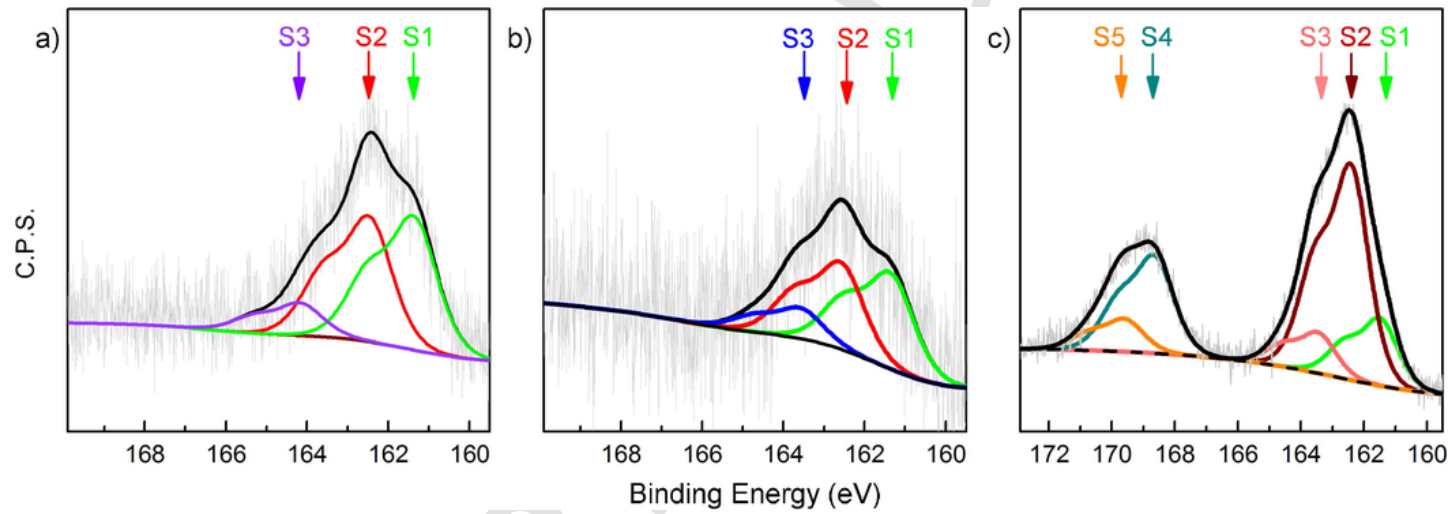

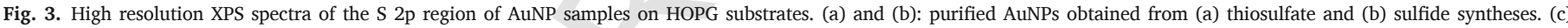
Non-arrested, non-purified AuNPs (thiosulfate synthesis). See the text for details.

[53-55]. The S1 component can be assigned to adsorbed sulfide (monomeric sulfur) on gold, while S2 is related to adsorbed polysulfide species and S3 to elemental S and probably to some physisorbed polysulfide species [53]. The total reduced $\mathrm{S}$ to $\mathrm{Au}$ intensity ratio $\left(\mathrm{S}_{\text {red }} / \mathrm{Au}\right)$, corrected for the relative sensitivity factors (RSF), is 0.13 , a value that is somehow larger than for S layers on $\mathrm{Au}(111)$ surfaces [54,55], as expected due to the curvature and the presence of defects in AuNPs.

Remarkably, our XPS spectra do not show evidences of adsorbed thiosulfate $[56,57]$ or any other oxidized sulfur species (sulfites, sulfates), which would appear at $\mathrm{BE} \geq 167 \mathrm{eV}[56,58]$. Therefore, XPS measurements of the purified AuNP samples unambiguously show that only reduced sulfur species are adsorbed on the AuNP surface. Similar results have been found in the case of AuNP samples obtained from the synthesis with sulfide and prepared in the same way as the above mentioned (Fig. 3b). This result is in sharp contrast with those previously reported by Pelaz et al. [24], whose S 2p XPS spectra showed a large doublet assigned to sulfate species (and only a very small peak in the region of reduced S species).

We have also measured the XPS spectra of samples prepared by drop casting AuNP dispersions that were not arrested and not purified, and without any water rinsing steps. As it can be seen in Fig. $3 c$, the region corresponding to reduced species can also be fitted with three components with S $2 \mathrm{p}_{3 / 2}$ binding energies $161.4 \mathrm{eV}$ (adsorbed monomeric S, S1), $162.4 \mathrm{eV}$ and $163.2 \mathrm{eV}$, the latter two most probably corresponding to different polysulfide species, both adsorbed and in solution [53]. The signal of elemental S (component S3 in Fig. 3a)) is probably masked by that of the polysulfides at approximately $163 \mathrm{eV}$. Most importantly, in this case the total reduced S to Au ratio value is significantly higher than that obtained for the purified AuNP samples (Fig. 4(a) and (b)). This supports the idea that the different polysulfide species are present not only on the surface of the AuNPs but also in the solution, and that they are mostly removed by centrifugation and also by rinsing the drop-casted substrates.

Moreover, in contrast to the spectra in 3(a) and 3(b), that in 3(c) shows the presence of two doublets that correspond to oxidized $\mathrm{S}$ species, with $\mathrm{S} 2 \mathrm{p}_{3 / 2} \mathrm{BE}=168.7$ and $169.6 \mathrm{eV}$, which can be assigned to sulfite and to sulfate species, respectively. Because of the use of a non-monochromatized source, these peaks contribute with satellites near $158 \mathrm{eV}$, which were taken into account but are not shown in Fig. 3c. Also, the peak at higher BE could have some contribution from the inner $S$ of some remaining thiosulfate species (outer $S$ would appear at about $162 \mathrm{eV}$ ). Again, the presence of various significant components assigned to $S$ reduced species ( Fig. 3c) disagrees with the simple reaction pathway previously proposed by other authors for similar syntheses and which consists of the direct oxidation of thiosulfate to sulfate and the reduction of $\mathrm{Au}(\mathrm{III})$ to $\mathrm{Au}(0)[24,27]$. 


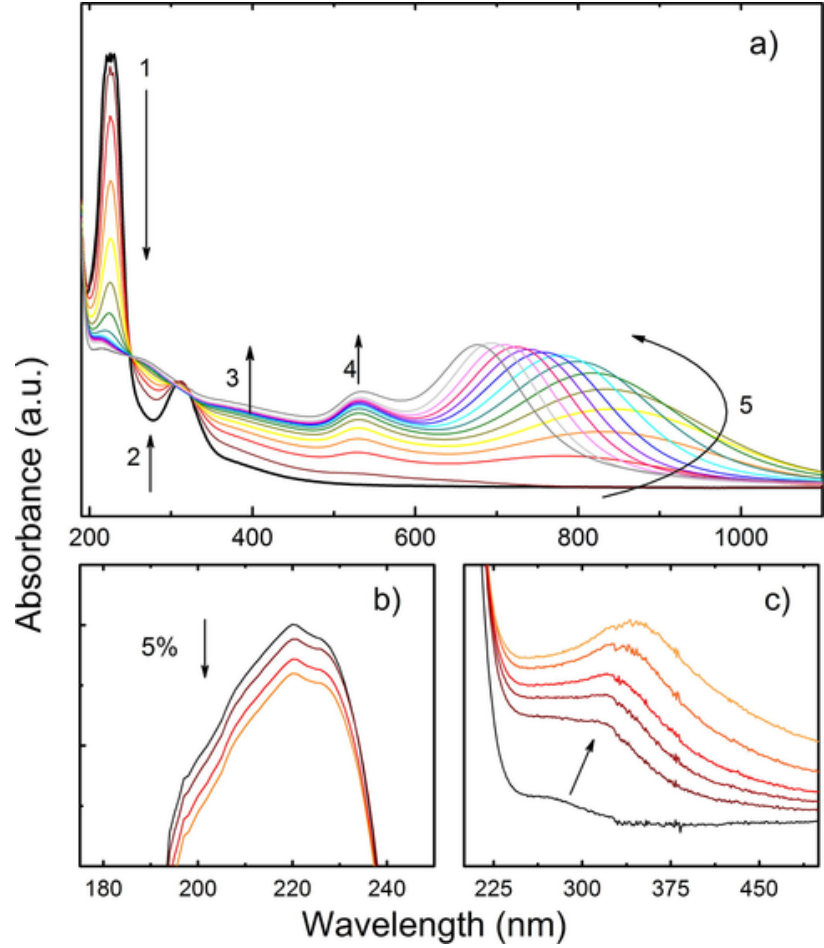

Fig. 4. (a) Absorbance spectra during the synthesis evolution. Arrow 1: decrease of the $\mathrm{Au}(\mathrm{III})$ peak; arrow 2: formation of a peak around $270 \mathrm{~nm}$, arrow 3: formation of a new peak, both of them bearing out the presence of polysulfides; arrows 4 and 5: evolution of the LSPR of the spherical and triangular AuNPs respectively. (b) Thiosulfate degradation in acidic solution. (c) Sulfide degradation in acidic solution to yield polysulfides and probably elemental sulfur. Time lapse corresponds to $1 \mathrm{~h}$.

\subsection{The possible chemical pathway}

In the case of the synthesis of AuNPs using sulfide as the reductant, it has been proposed that sulfide species $\left(\mathrm{HS}^{-}, \mathrm{H}_{2} \mathrm{~S}\right.$ ) reduce $\mathrm{Au}(\mathrm{III})$ to $\mathrm{Au}(0)$ [28]. Contrarily to what was previously proposed by other authors for similar syntheses [59], there was no evidence of the formation of any oxidized $S$ species which could act as the gold reductant, neither in solution nor in the surface of the AuNPs [33]. This knowledge is now useful to shed light on the possible reactions that take place in the case of the reduction with thiosulfate, which is clearly more difficult to interpret due to its complex chemistry in acidic aqueous solutions. The fact that the same reaction products are found for both syntheses leads us to think that reduced $\mathrm{S}$ species also play a key role in the thiosulfate synthesis.

The chemistry of thiosulfate in acidic media opens the possibility for alternative reaction pathways that can lead to a completely different chemistry. As mentioned before, thiosulfate salts decompose in acidic media to yield elemental sulfur, sulfur dioxide, hydrogen sulfide and polysulfanes, among others [34]. Moreover, it has been reported that decomposition of thiosulfate is accelerated in the presence of metallic ions, with formation of metal sulfides [35]. Also, when thiosulfate in excess reacts with $\mathrm{Au}$ (III) ions, $\mathrm{Au}\left(\mathrm{S}_{2} \mathrm{O}_{3}\right)_{2}{ }^{3-}$ complexes are formed that can decompose to yield sulfides on solid surfaces, and small AuNPs can be obtained upon heat treatment at $300^{\circ} \mathrm{C}$ [60].

In Fig. 4a we show successive UV-vis-NIR spectra recorded in the $190-1100 \mathrm{~nm}$ range as the synthesis evolves; the time lapse between the first and the last spectrum is $60 \mathrm{~min}$. The most noticeable changes, as the reaction proceeds, are the disappearance of the large peaks at 227 and $313 \mathrm{~nm}$ belonging to $\mathrm{HAuCl}_{4}$ (the former also has a contribution from thiosulfate at $216 \mathrm{~nm}$; see Fig.4b) and the appearance of the above mentioned LSPR peaks at approximately $530 \mathrm{~nm}$ and in the NIR. To assess the possible degradation of thiosulfate due to the high acidity of the reaction mixture $\left(\mathrm{pH}=2-3\right.$ ), we followed the peak of $\mathrm{S}_{2} \mathrm{O}_{3}{ }^{2-}$ at $216 \mathrm{~nm}$ in a medium free of gold for the duration of the synthesis (see Fig. 4b). We have found that in the considered time lapse the reagent is not appreciably decomposed in the absence of Au(III) ions, as the intensity of the peak only decreases a $5 \%$ after a time lapse similar to that of the whole synthesis process.

Next, we have carefully analyzed the successive spectra in Fig. 4a to try to detect any $S$ species that are of interest in view of the XANES and XPS results. As the synthesis proceeds, some small peaks and humps appear in the $280-400 \mathrm{~nm}$ region which can be attributed to both the formation of polysulfide species and elemental $S$ [61,62]. In Fig. 4c similar peaks can be observed in a non-deaerated sulfide solution, prepared at the same $\mathrm{pH}$ of the reaction mixture, in the absence of gold ions. The presence of sulfide species, however, cannot be detected by UV-vis-NIR spectroscopy, as sulfide bears a peak at $230 \mathrm{~nm}$ that is completely masked by the large peak at $227 \mathrm{~nm}\left(\mathrm{HAuCl}_{4}\right)$ and that at $216 \mathrm{~nm}$ (thiosulfate). Moreover, this peak decreases with decreasing $\mathrm{pH}$ values because the species that absorbs is $\mathrm{HS}^{-}$and not $\mathrm{H}_{2} \mathrm{~S}$, and the speciation diagram of sulfide shows that at $\mathrm{pH} 2-3$ the predominant species is $\mathrm{H}_{2} \mathrm{~S}$ [63-65].

During the first stages of the reaction, $\mathrm{S}_{2} \mathrm{O}_{3}{ }^{2-}$ ions could reduce $\mathrm{Au}(\mathrm{III})$ to $\mathrm{Au}(\mathrm{I})$ forming $\mathrm{Au}\left(\mathrm{S}_{2} \mathrm{O}_{3}\right)_{2}{ }^{3-}$ complexes; however, we could not detect this thiosulfate complex, which shows a peak at $203 \mathrm{~nm}$ [66]. This could be attributed
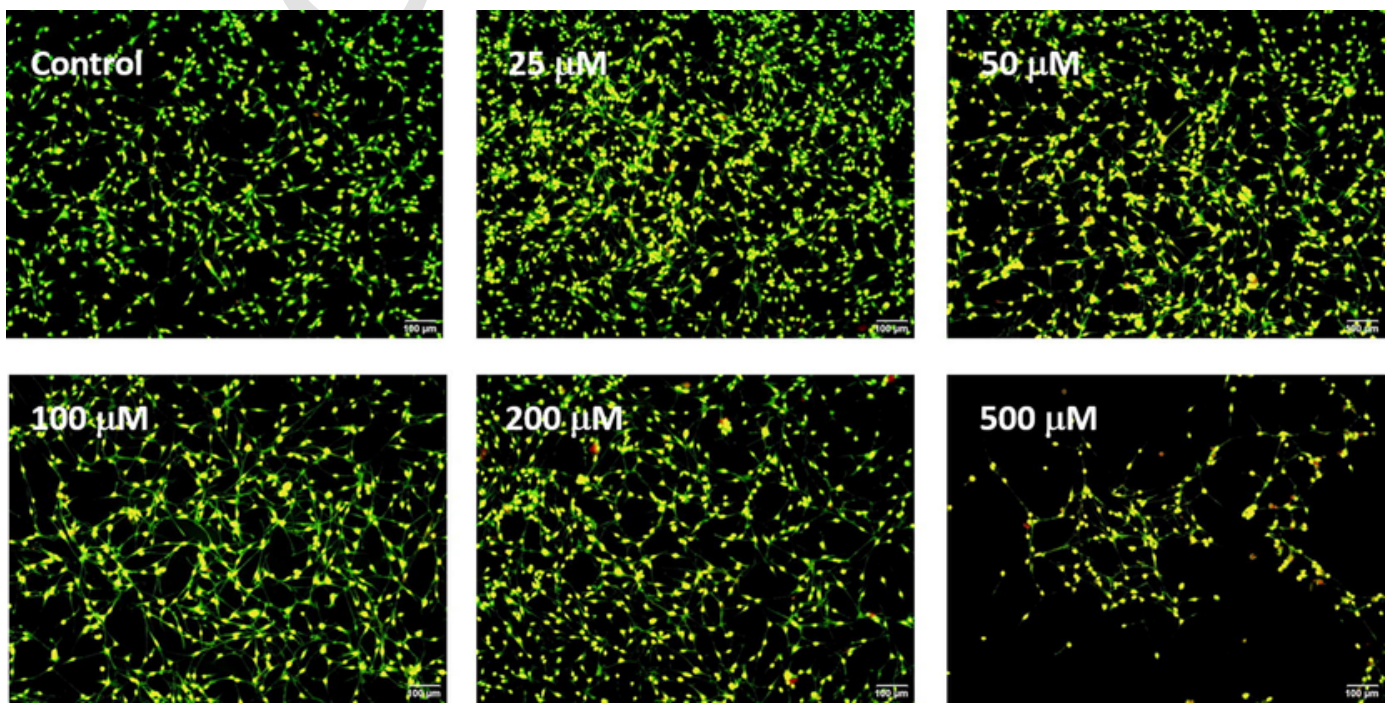

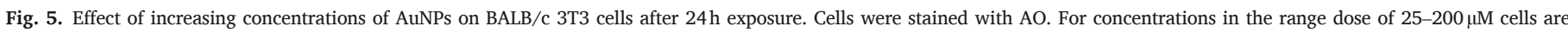
indistinguishable from control. For the $500 \mu \mathrm{M}(\approx 100 \mu \mathrm{g} / \mathrm{mL})$ concentration cells are clearly affected. 
to the fact that the complex is more stable in alkaline oxidant media, whereas in acidic media it decomposes into sulfide species $[60,66]$. Moreover, $\mathrm{Au}\left(\mathrm{S}_{2} \mathrm{O}_{3}\right)_{2}{ }^{3-}$ has a lower solution stability with respect to another well-known $\mathrm{Au}(\mathrm{I})$ complex, $\mathrm{Au}(\mathrm{SH})_{2}$, which is more stable in neutral or mild acidic media $[67,68]$. Other oxidized species that are usual products of the disproportionation of thiosulfate, like sulfate and sulfite, do not show peaks in the studied wavelength range [62].

Based on the combined experimental evidence from XPS, XANES and UV-vis-NIR spectroscopy, and on previous results from other authors, we propose that, as the synthesis takes place in acidic condition, some $\mathrm{S}_{2} \mathrm{O}_{3}{ }^{2-}$ ions in the solution disproportionate to yield (a) sulfide species (mainly $\mathrm{H}_{2} \mathrm{~S}$ and also $\mathrm{HS}^{-}$) and some elemental S, and (b) sulfate and sulfite ions (see Scheme 1) [35,69-71]. For instance, Pryor has studied the disproportionation of thiosulfate to sulfide and sulfate at $250-280^{\circ} \mathrm{C}$ [71], while other authors have also reported that thiosulfate reduces to sulfide in milder conditions [35,36]. Also, $\mathrm{S}_{2} \mathrm{O}_{3}{ }^{2-}$ bond cleavage yielding reduced $\mathrm{S}$ species and $\mathrm{SO}_{3}{ }^{2-}$ species have been reported for alkyl thiosulfate on Au surfaces [57]. Moreover, some bacteria produce the anaerobic disproportionation of thiosulfate to sulfate and sulfide, with elemental sulfur and sulfite probably being the main reaction intermediates [72].

In our case, we suggest that the decomposition of thiosulfate to sulfide (and $\mathrm{SO}_{4}{ }^{2-} / \mathrm{SO}_{3}{ }^{2-}$ ), which does not appreciably occur at ambient temperature, is favored in the presence of metallic ions: in fact, sulfide ions are consumed in the reduction of $\mathrm{Au}(\mathrm{III})$ ions to metallic gold and thus the disproportionation reaction is displaced towards the products. As for the other reduced S species detected, elemental S can be a product of the reduction of $\mathrm{Au}(\mathrm{III})$ by sulfide species [28] and can also be formed from the disproportionation of thiosulfate in acidic media [70], while polysulfides are usually formed by reaction of elemental $\mathrm{S}$ with sulfide species [73]. Also, sulfide oxidation by dissolved oxygen in the reaction mixture can contribute to the formation of polysulfides and elemental S.

Therefore, while alternative routes, like the direct oxidation of thiosulfate to sulfite or sulfate upon gold reduction cannot be discarded, the synthesis of AuNTs with thiosulfate as reductant clearly involves the reduction of $\mathrm{Au}(\mathrm{III})$ ions via reduced $\mathrm{S}$ species. In fact, the same reaction products as in the sulfide synthesis (i.e., a mixture of sulfides, polysulfides and elemental S) are formed and these determine the surface chemistry of the AuNPs. The sulfide and polysulfide species are strongly adsorbed on the AuNTs (and spherical AuNPs) and this will determine their surface properties and also the possible interactions at the nano-bio interface [25,42-44,74].

As a result, the possibility of developing applications based on these AuNTs will be somehow more difficult if postfunctionalization steps based on ligand exchange with thiols are necessary $[25,42]$. In this sense, it will be important to develop methods to remove the adsorbed reduced $\mathrm{S}$ species, possibly by oxidation, in order to have more labile adsorbates (e.g., oxidized $\mathrm{S}$ species such as sulfate and sulfite) that can be exchanged by thiol-bearing molecules having a higher affinity for the gold surface.

\subsection{Cell culture assays}

It is well known that $\mathrm{H}_{2} \mathrm{~S}$ inhibits some enzymes, such as cytochrome $c$ oxidase, the terminal electron acceptor of the mitochondrial electron transport chain $[44,75]$. The effect of this molecule on the cell physiology

$$
\text { is }
$$
still not clear: while some authors are investigating the beneficial effects of this molecule some others see it as a toxic agent $[43,74]$. At this point, in view of the promising application of these AuNTs for plasmonic photothermal therapies, and the fact that the sulfide species present could interfere with enzymatic reactions, it is important to assess the effect of the S-capped nanoparticles (AuNTs and spherical AuNPs) on mammalian cells.

To this end, we have studied the effects on BALB/c 3T3 cells of different concentrations of purified AuNPs (expressed as final Au concentrations). Fig. 5 shows representative microscopic images of cell viability by AO staining for cell cultures treated with different AuNP concentrations. The analysis of cell coverage for each AuNP concentration with respect to cell control (cultures without AuNPs) after $24 \mathrm{~h}$ revealed similar results for the $25-200 \mu \mathrm{M}$ range dose, with minimal variations (c.a. $95 \%)$. We found a significant $(\mathrm{p}<0.001)$ decrease in coverage values, close to $25(2) \%$ only for the maximum tested dose $(500 \mu \mathrm{M} \approx 100 \mu \mathrm{g} /$ $\mathrm{mL})$. These values are in accordance with previous reports that show similar results for these AuNP concentrations [11,24-26,76,77].

In addition, the effect of the treatment with AuNPs on BALB/c 3T3 cells during $24 \mathrm{~h}$ evaluated from the NR assay is summarized in Fig. 6. No significant differences with respect to the control were found in cells treated with $25,50,100$ or $200 \mu \mathrm{M}$ concentrations, but a slight decrease in the lysosomal activity in cultures with high AuNPs concentration $(500 \mu \mathrm{M})$ was observed. Similar results were found for the mitochondrial activity evaluated using MTT test after $24 \mathrm{~h}$ cell exposure to AuNPs in the 25-500 $\mu \mathrm{M}$ range. However, in the case of the $500 \mu \mathrm{M}$ dose there is a significant difference $(\mathrm{p}<0.001)$ with respect to the control measurements (Fig. 6).

For all the performed assays cell viability is not diminished for concentrations $\leq 200 \mu \mathrm{M}$, and this is achieved without any surface postfunctionalization, such as PEGylation, that would increase AuNP biocompatibility. It is worth to mention that $200 \mu \mathrm{M}$ is a much higher concentration than those used for in vivo assays. For instance, De la Fuente et al have previously reported typical doses of $100 \mu \mathrm{L}$ of a $1 \mathrm{mg} / \mathrm{mL}$ dispersion for mouse administration [76,78] in studies whose purpose is to test the efficiency of the photothermal therapy. However, even if these results are promising for the use of these AuNPs for biomedical applications, it would still be desirable to remove the S adlayer without changing the structure of the AuNTs (which defines their optical properties) [21].

\section{Conclusions}

In this work we have made a detailed study of the AuNPs formed by reduction of $\mathrm{Au}(\mathrm{III})$ with thiosulfate ions by means of UV-vis-NIR spectroscopy, XPS, XANES, HRTEM and AFM. This synthesis gives a mixture of

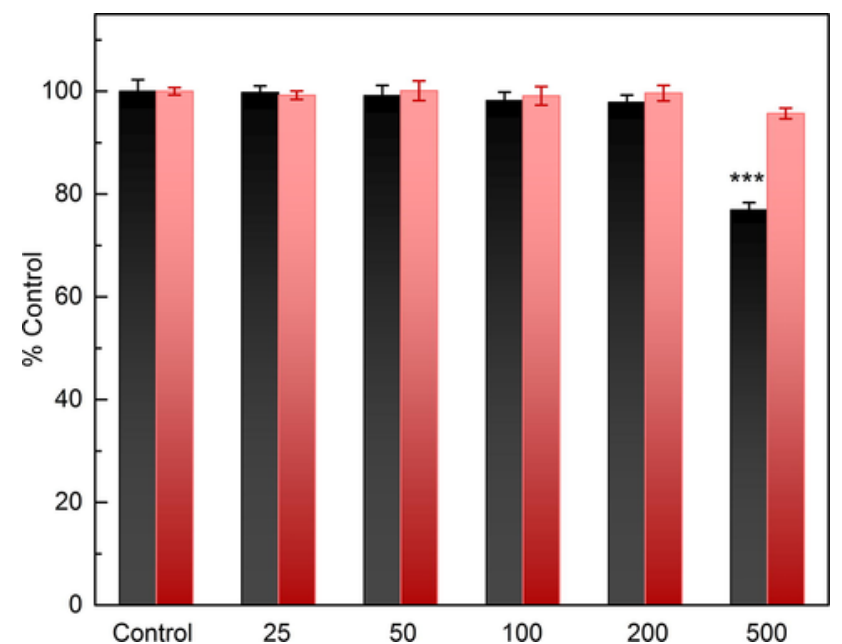

Fig. 6. Cellular response to different concentrations of AuNPs after $24 \mathrm{~h}$ exposure as evaluated by MTT assay (black bars) and NR uptake (red bars). Error bars: standard error of the mean value. ${ }^{* * *}$ Significant difference at $\mathrm{p}<0.001$. (For interpretation of the references to color in this figure legend, the reader is referred to the web version of this article.) 
like AuNPs and AuNTs that absorb in the NIR-I window, an optimal region for photothermal applications. The experimental evidence presented allows us to conclude that the synthesis does not simply proceed via the oxidation of thiosulfate to sulfate species as the $\mathrm{Au}(\mathrm{III})$ ions reduce to metallic gold [24,27]. Instead, while this route cannot be precluded, there is a more important pathway that involves thiosulfate decomposition to sulfide species and the oxidation of these species to yield elemental S and polysulfides, all of which adsorb on the surface of the AuNPs. We have therefore demonstrated that, unlike what was previously reported for similar syntheses, AuNTs (and spherical AuNPs) are not covered by weakly physisorbed sulfate ions that could be easily removed from the gold surface [24,27], but instead are covered by the reduced $\mathrm{S}$ species, which are strongly bonded. To fully characterize this product is a crucial point in order to optimize the use of the AuNTs for any application. It is clear that the presence of these species implies possible non-desired secondary effects, as the sulfide can interfere with different enzymatic reactions. Thus, the knowledge that these particles are covered by reduced S species has to be taken into account.

In order to assess the effect of the S-capped AuNPs on mammalian cells we have studied, as a first step, their effect on BALB/c 3T3 cells at different concentrations. Even if the AuNPs were not functionalized with an additional biocompatible adlayer, it can be concluded that they do not show a detrimental effect on cell viability for concentrations as high as $200 \mu \mathrm{M}(\approx 40 \mu \mathrm{g} / \mathrm{L})$, which is higher than the doses used for in vivo assays $[76,78]$.

However, regarding possible postfunctionalization designs, it would still be desirable to remove the $S$ adlayer without changing the structure of the AuNTs. This issue is of particular importance for further postfunctionalization steps based on the chemistry of thiols, as these are usually performed by ligand exchange methods. This is relevant for instance to increase AuNP bioavailability, or to combine photothermal therapy with the release of a drug induced by NIR laser irradiation. Indeed, more studies of the interfacial properties of these AuNPs will be necessary, including the understanding of morphological and chemical changes resulting from the steps needed to obtain functionalized separate AuNTs for the applications.

\section{Acknowledgements}

This work has been financially supported by ANPCyT (PICT 2012-0836, 2010-2554, 2012-1136 and 2015-2285), CONICET (PIP 112-201201-00093 and 112-201101-01035) and UNLP. Partial support by Laboratório Nacional de Luz Síncrotron (LNLS) under proposals SXS 20150180 and XAFS2 20160225 is also acknowledged. The authors wish to thank Dr. Magdalena Gherardi from the Instituto de Investigaciones Biomédicas en Retrovirus y SIDA (INBIRS), Facultad de Medicina, Universidad de Buenos Aires, Argentina for kindly providing the BALB/ c 3T3 cell line.

\section{Appendix A. Supplementary material}

Supplementary data associated with this article can be found, in the online version, at https://doi.org/10.1016/j.apsusc.2018.09.009.

\section{References}

[1] P.K. Jain, et al., Calculated absorption and scattering properties of gold nanoparticles of different size, shape, and composition: applications in biological imaging and biomedicine, J. Phys. Chem. B 110 (14) (2006) 7238-7248.

[2] K.M. Mayer, J.H. Hafner, Localized surface plasmon resonance sensors, Chem. Rev. 111 (6) (2011) 3828-3857.

[3] E. Pensa, et al., The chemistry of the sulfur-gold interface: in search of a unified model, Acc. Chem. Res. 45 (8) (2012) 1183-1192.

[4] T.K. Sau, et al., Properties and applications of colloidal nonspherical noble metal nanoparticles, Adv. Mater. 22 (16) (2010) 1805-1825.

[5] M.I. Stockman, Nanoplasmonic sensing and detection, Sci.-Appl. Opt. 348 (6232) (2015) 287-288.

[6] A.I. Henry, et al., Correlated structure and optical property studies of plasmonic nanoparticles, J. Phys. Chem. C 115 (19) (2011) 9291-9305.

[7] L. Chen, et al., High-yield seedless synthesis of triangular gold nanoplates through oxidative etching, Nano Lett. 14 (12) (2014) 7201-7206.

[8] J.E. Millstone, et al., Observation of a quadrupole plasmon mode for a colloidal solution of gold nanoprisms, J. Am. Chem. Soc. 127 (15) (2005) 5312-5313.
[9] L. Scarabelli, et al., Monodisperse gold nanotriangles: size control, large-scale self-assembly, and performance in surface-enhanced Raman scattering, Acs Nano 8 (6) (2014) 5833-5842.

[10] Y.M. Zhai, et al., Polyvinylpyrrolidone-induced anisotropic growth of gold nanoprisms in plasmon-driven synthesis, Nat. Mater. 15 (8) (2016) 889.

[11] G. Alfranca, et al., Gold nanoprism-nanorod face off: comparing the heating efficiency, cellular internalization and thermoablation capacity, Nanomedicine (Lond.) 11 (22) (2016) 2903-2916.

[12] A. Sabur, M. Havel, Y. Gogotsi, SERS intensity optimization by controlling the size and shape of faceted gold nanoparticles, J. Raman Spectrosc. 39 (1) (2008) 61-67.

[13] L. Soares, et al., Localized surface plasmon resonance (LSPR) biosensing using gold nanotriangles: detection of DNA hybridization events at room temperature, Analyst 139 (19) (2014) 4964-4973.

[14] K. Nambara, et al., Reverse size dependences of the cellular uptake of triangular and spherical gold nanoparticles, Langmuir 32 (47) (2016) 12559-12567.

[15] M. Kasture, M. Sastry, L.V. Prasad, Halide ion controlled shape dependent gold nanoparticle synthesis with tryptophan as reducing agent: enhanced fluorescent properties and white light emission, Chem. Phys. Lett. 484 (4-6) (2010) 271-275.

[16] S.P. Chandran, et al., Synthesis of gold nanotriangles and silver nanoparticles using aloevera plant extract, Biotechnol. Prog. 22 (2) (2008) 577-583.

[17] S.S. Shankar, et al., Controlling the optical properties of lemongrass extract synthesized gold nanotriangles and potential application in infrared-absorbing optical coatings, Chem. Mater. 17 (3) (2005) 566-572.

[18] S.S. Shankar, et al., Biological synthesis of triangular gold nanoprisms, Nat. Mater. 3 (2004) 482.

[19] F. Liebig, et al., "Green" gold nanotriangles: synthesis, purification by polyelectrolyte/micelle depletion flocculation and performance in surface-enhanced Raman scattering, RSC Adv. 6 (40) (2016) 33561-33568.

[20] F. Liebig, A.F. Thünemann, J. Koetz, Ostwald ripening growth mechanism of gold nanotriangles in vesicular template phases, Langmuir 32 (42) (2016) 10928-10935.

[21] M.A. Huergo, et al., Optical nanoparticle sorting elucidates synthesis of plasmonic nanotriangles, Acs Nano 10 (3) (2016) 3614-3621.

[22] J.J. Diao, H. Chen, Near infrared surface plasmon resonance of gold tabular nanostructures in the HAuCl4-Na2S reaction, J. Chem. Phys. 124 (11) (2006) 116103

[23] Y. Mikhlin, et al., Formation of gold and gold sulfide nanoparticles and mesoscale intermediate structures in the reactions of aqueous $\mathrm{HAuCl} 4$ with sulfide and citrate ions, Phys. Chem. Chem. Phys. 11 (26) (2009) 5445-5454.

[24] B. Pelaz, et al., Tailoring the synthesis and heating ability of gold nanoprisms for bioapplications, Langmuir 28 (24) (2012) 8965-8970.

[25] S.-E. Saverot, et al., Characterization of conventional one-step sodium thiosulfate facilitated gold nanoparticle synthesis, Nanoscale Res. Lett. 10 (1) (2015) 241.

[26] Y. Yang, et al., Human CIK cells loaded with Au nanorods as a theranostic platform for targeted photoacoustic imaging and enhanced immunotherapy and photothermal therapy, Nanoscale Res. Lett. 11 (1) (2016) 285.

[27] G. Zhang, et al., Tunability and stability of gold nanoparticles obtained from chloroauric acid and sodium thiosulfate reaction, Nanoscale Res. Lett. 7 (1) (2012) 337.

[28] R.D. Averitt, D. Sarkar, N.J. Halas, Plasmon resonance shifts of Au-coated Au2S nanoshells: insight into multicomponent nanoparticle growth, Phys. Rev. Lett. 78 (22) (1997) 4217-4220.

[29] A.M. Gobin, et al., Near-infrared-resonant gold/gold sulfide nanoparticles as a photothermal cancer therapeutic agent, Small 6 (6) (2010) 745-752.

[30] T.J. Norman, et al., Near infrared optical absorption of gold nanoparticle aggregates, J. Phys. Chem. B 106 (28) (2002) 7005-7012.

[31] L. Ren, G.M. Chow, Synthesis of nir-sensitive Au-Au2S nanocolloids for drug delivery, Mater. Sci. Eng. C-Biomimet. Supramol. Syst. 23 (1-2) (2003) 113-116.

[32] H.S. Zhou, et al., Controlled synthesis and quantum-size effect in gold-coated nanoparticles, Phys. Rev. B 50 (16) (1994) 12052-12056.

[33] M.A. Huergo, et al., New insight into the chemical nature of the plasmonic nanostructures synthesized by the reduction of Au(III) with sulfide species, Langmuir 33 (27) (2017) 6785-6793.

[34] P.W. Atkins, T. Overton, J.P. Rourke, M.T. Wellet, F.A. Armstrong, Shriver and Atkins' Inorganic Chemistry, fifth ed., Oxford University Press, Oxford: Great Britain, 2010.

[35] R. Woods, et al., A spectroelectrochemical study of surface species formed in the gold/thiosulfate system, J. Electrochem. Soc. 153 (7) (2006) D105-D113.

[36] M.D. Lay, K. Varazo, J.L. Stickney, Formation of sulfur atomic layers on gold from aqueous solutions of sulfide and thiosulfate: studies using EC-STM, UHV-EC, and TLEC, Langmuir 19 (20) (2003) 8416-8427.

[37] J.C. Azcárate, et al., Understanding the surface chemistry of thiolate-protected metallic nanoparticles, J. Phys. Chem. Lett. 4 (18) (2013) 3127-3138.

[38] R. Dinkel, B. Braunschweig, W. Peukert, Fast and slow ligand exchange at the surface of colloidal gold nanoparticles, J. Phys. Chem. C 120 (3) (2016) 1673-1682.

[39] C.Y. Wu, et al., High-spatial-resolution mapping of catalytic reactions on single particles, Nature 541 (7638) (2017) 511-515.

[40] A.M. Alkilany, S.E. Lohse, C.J. Murphy, The gold standard: gold nanoparticle libraries to understand the nano-bio interface, Acc. Chem. Res. 46 (3) (2013) $650-661$.

[41] S.T. Kim, et al., The role of surface functionality in determining nanoparticle cytotoxicity, Acc. Chem. Res. 46 (3) (2013) 681-691.

[42] A.E. Nel, et al., Understanding biophysicochemical interactions at the nano-bio in terface, Nat. Mater. 8 (7) (2009) 543-557.

[43] J.J. Jiang, et al., Hydrogen sulfide-mechanisms of toxicity and development of an antidote, Sci. Rep. (2016) 6.

[44] R. Wever, B. van Gelder, D.V. Dervartanian, Biochemical and biophysical studies on cytochrome c oxidase. XX. Reaction with sulphide, Biochim. Biophys. Acta 387 (2) (1975) 189-193. 
[48] E. Borenfreund, J.A. Puerner, Toxicity determined in vitro by morphological alterations and neutral red absorption, Toxicol. Lett. 24 (2-3) (1985) 119-124.

[49] T. Mosmann, Rapid colorimetric assay for cellular growth and survival: application to proliferation and cytotoxicity assays, J. Immunol. Methods 65 (1-2) (1983) 55-63.

[50] P.R. Twentyman, M. Luscombe, A study of some variables in a tetrazolium dye (Mtt) based assay for cell-growth and chemosensitivity, Br. J. Cancer 56 (3) (1987) 279-285.

[51] A.I. Kirkland, et al., Structural studies of trigonal lamellar particles of gold and silver, Proc. Roy. Soc. London Ser. a-Math. Phys Eng. Sci. 440 (1910) (1993) 589-609.

[52] A. Vairavamurthy, Oxidation-state of sulfur in thiosulfate and implications for anaerobic energy-metabolism, Geochim. Cosmochim. Acta 57 (13) (1993), 3199-3199.

[53] M. Fantauzzi, et al., Exploiting XPS for the identification of sulfides and polysulfides, Rsc Adv. 5 (93) (2015) 75953-75963.

[54] F. Lobo Maza, et al., New insight into the interface chemistry and stability of glutathione self-assembled monolayers on Au(111), J. Phys. Chem. C 120 (27) (2016) 14597-14607.

[55] P.G. Lustemberg, et al., Spontaneously formed sulfur adlayers on gold in electrolyte solutions: adsorbed sulfur or gold sulfide?, J. Phys. Chem. C 112 (30) (2008) 11394-11402.

[56] B.J. Lindberg, et al., Molecular spectroscopy by means of ESCA II. Sulfur compounds. Correlation of electron binding energy with structure, Physica Scr. 1 (5-6) (1970) 286.

[57] J. Lukkari, et al., Organic thiosulfates (Bunte salts): novel surface-active sulfur compounds for the preparation of self-assembled monolayers on gold, Langmuir 15 (10) (1999) 3529-3537.

[58] M. Fantauzzi, et al., A contribution to the surface characterization of alkali metal sulfates, J. Electron Spectrosc. Relat. Phenom. 193 (2014) 6-15.

[59] A.M. Schwartzberg, et al., Reduction of $\mathrm{HAuCl} 4$ by Na2S revisited: the case for $\mathrm{Au}$ nanoparticle aggregates and against Au2S/Au core/shell particles, J. Phys. Chem. C 111 (25) (2007) 8892-8901.

[60] B. Tian, et al., Preparation of $\mathrm{Au} / \mathrm{TiO} 2$ catalysts from $\mathrm{Au}(\mathrm{I})$-thiosulfate complex and study of their photocatalytic activity for the degradation of methyl orange, Appl. Catal. B 79 (4) (2008) 394-401.

[61] N.G. Heatley, E.J. Page, Estimation of elemental sulfur by ultraviolet absorption, Anal. Chem. 24 (11) (1952), 1854-1854.

[62] S.A. Khan, UV-ATR spectroscopy study of the speciation in aqueous polysulfide electrolyte solutions, Int. J. Electrochem. Sci. 7 (1) (2012) 561-568.

[63] O. Thomas, B. Roig, Chapter 5 Mineral constituents, in: O. Thomas, C. Burgess (Eds.), UV-Visible Spectrophotometry of Water and Wastewater, Elsevier, 2007, pp. 163-195.
[64] D. Harris, Quantitative Chemical Analysis, eighth ed., Freeman and Company, New York, 2010.

[65] E.A. Guenther, K.S. Johnson, K.H. Coale, Direct ultraviolet spectrophotometric determination of total sulfide and iodide in natural waters, Anal. Chem. 73 (14) (2001) 3481-3487.

[66] M.I. Jeffrey, S.D. Brunt, The quantification of thiosulfate and polythionates in gold leach solutions and on anion exchange resins, Hydrometallurgy 89 (1-2) (2007) 52-60.

[67] R.A. Bryce, et al., EXAFS and density functional study of gold(I) thiosulfate complex in aqueous solution, J. Phys. Chem. A 107 (14) (2003) 2516-2523.

[68] D. Rickard, G.W. Luther, Metal sulfide complexes and clusters, Sulfide Mineral. Geochem. 61 (2006) 421-504.

[69] M.G. Aylmore, D.M. Muir, Thiosulfate leaching of gold - a review, Miner. Eng. 14 (2) (2001) 135-174.

[70] F.A. Cotton, G. Wilkinson, C.A. Murillo, M. Bochmann, Advanced Inorganic Chemistry: A Comprehensive Text, sixth ed., Wiley-Interscience, New York, 1999.

[71] W.A. Pryor, The kinetics of the disproportionation of sodium thiosulfate to sodium sulfide and sulfate, J. Am. Chem. Soc. 82 (18) (1960) 4794-4797.

[72] H. Cypionka, A.M. Smock, M.E. Böttcher, A combined pathway of sulfur compound disproportionation in Desulfovibrio desulfuricans, FEMS Microbiol. Lett. 166 (2) (1998) 181-186.

[73] A.N. Buckley, I.C. Hamilton, R. Woods, An Investigation of the Sulfur(2)/Sulfur(0) System on Gold Electrodes, vol. 216, 1987, pp. 213-227.

[74] B. Szczesny, et al., Inhibition of hydrogen sulfide biosynthesis sensitizes lung adenocarcinoma to chemotherapeutic drugs by inhibiting mitochondrial DNA repair and suppressing cellular bioenergetics, Sci. Rep. (2016) 6.

[75] C.E. Cooper, G.C. Brown, The inhibition of mitochondrial cytochrome oxidase by the gases carbon monoxide, nitric oxide, hydrogen cyanide and hydrogen sulfide: chemical mechanism and physiological significance, J. Bioenerget. Biomembr. 40 (5) (2008) 533-539

[76] C.C. Bao, et al., Gold nanoprisms as a hybrid in vivo cancer theranostic platform for in situ photoacoustic imaging, angiography, and localized hyperthermia, Nano Res. 9 (4) (2016) 1043-1056.

[77] Y. Li, et al., Infrared light-absorbing gold/gold sulfide nanoparticles induce cell death in esophageal adenocarcinoma, Int. J. Nanomed. 8 (2013) 2153-2161.

[78] J.S. Han, et al., Glucose-functionalized Au nanoprisms for optoacoustic imaging and near-infrared photothermal therapy (vol 8, pg 492, 2016), Nanoscale 8 (3) (2016), 1704-1704. 